\title{
BOKASHI E COMPRIMENTO DE ESTACAS NA PROPAGAÇÃO VEGETATIVA DE Pereskia aculeata Plum (ORA-PRO-NÓBIS)
}

\author{
BOKASHI AND LENGTH OF CUTTINGS IN THE VEGETATIVE PROPAGATION OF \\ Pereskia aculeata Plum (ORA-PRO-NÓBIS) \&
}

BOKASHI Y ALTURA DE ESTACAS EN LA PROPAGACIÓN VEGETATIVA DE Pereskia aculeata Plum (ORA-PRO-NÓBIS) 8

Recebido em: 11/11/2020 - Aprovado em: 02/12/2020 - Publicado em: 20/04/2021

Juliana Milene Silverio' (juliana.milene@hotmail.com)

Cleberton Correia Santos ${ }^{1}$ (cleber_frs@yahoo.com.br)

Venâncio Estevão Wilkomm² (venancio.wilkomm@hotmail.com)

Diogo Roberto Bruschis Karas¹ (diogorbkaras@gmail.com)

Maria do Carmo Vieira1 (mariavieira@ufgd.edu.br)

Néstor Antonio Heredia Zárate' (nestorzarate@ufgd.edu.br)

1 Universidade Federal da Grande Dourados (UFGD), Dourados, MS, Brasil.

2 Centro Universitário da Grande Dourados (UNIGRAN), Dourados, MS, Brasil.

\section{RESUMO}

Existem poucas informações técnicas sobre os fatores que podem influenciar a propagação vegetativa de Pereskia aculeata Plum. Assim, objetivou-se com este estudo conhecer o efeito do comprimento de estacas e uso de bokashi na propagação de $P$. aculeata. Avaliaram-se três comprimentos de estacas: 15, 20 e $25 \mathrm{~cm}$, sem e com bokashi, sob viveiro com $50 \%$ de sombreamento. As estacas foram propagadas em recipientes plásticos de $500 \mathrm{~mL}$, com substrato Latossolo Vermelho Distroférrico + Tropstrato ${ }^{\circledR}(1: 1$, v/v). As brotações foram avaliadas semanalmente após o dia da primeira brotação, e aos 60 dias após a estaquia, fez-se a colheita das plântulas inteiras. A maior porcentagem de brotação foi de $60 \%$ e o índice de velocidade de brotação de 0,8737 nas mudas provenientes de estacas de $20 \mathrm{~cm}$ com bokashi. $O$ maior número de brotos $(6,12)$ e de folhas $(24,83)$ foi constatado nas estacas de $25 \mathrm{~cm}$, independente do bokashi. Os maiores valores de comprimento das raízes $(16,58 \mathrm{~cm})$ e de produção de massas frescas e secas de brotações $(19,23$ e 11,22 $\mathrm{g}$, respectivamente) foram nas estacas de $25 \mathrm{~cm}$. A adição de bokashi em estacas de $25 \mathrm{~cm}$ favoreceu maior produção de massa seca de brotações. O uso de estacas de $25 \mathrm{~cm}$ contribuiu na produção de mudas de $P$. aculeata por favorecer os indicadores de brotação, área foliar e índices fisiológicos.

Keywords: Biofertilizante. Brotação. Estaquia. Planta medicinal. PANC's. 


\section{INTRODUÇÃO}

Dentre as espécies de interesse agromedicinal, a Ora-pro-nóbis (Pereskia aculeata Plum, Cactaceae) é uma planta alimentícia não convencional (PANC). As folhas da espécie são carnosas, podendo ser consumidas in natura em saladas e pratos cozidos devido às elevadas concentrações de proteína, cálcio, ferro e fibras (ALMEIDA et al., 2012; KINUPP \& LORENZI, 2014).

Quanto às atividades biológicas da planta, constatou-se que as folhas de $P$. aculeata apresentam potencial como fontes de compostos antioxidantes (SOUZA et al., 2014), e que ao serem adicionadas em dieta hipercalórica reduziram as concentrações de triglicerídeos em ratos (SOUZA et al., 2015). Assim, sua propagação visando o cultivo para uso medicinal e alimentício tem elevada importância, sendo uma alternativa de geração de renda para o produtor rural (SOUZA et al., 2016) e diversificação nos agroecossistemas.

A propagação de $P$. aculeata pode ser sexuada; porém, as sementes são de difícil obtenção por apresentarem desuniformidade na germinação e emergência, sendo necessário o aprimoramento de métodos visando à obtenção de mudas. A propagação vegetativa por meio da estaquia tem sido considerada uma alternativa promissora e difundida, pois possibilita padronização genética, baixo custo e tempo reduzido de juvenilidade (SILVA et al., 2015), além de ser de fácil execução.

Fatores relacionados à estaca podem influenciar na propagação das plantas, dentre eles pode-se citar o comprimento. Isso porque o tamanho das estacas está interligado à condição nutricional, quantidade de reservas e ao número de gemas (PIZZATO et al., 2011), sendo uma importante característica a ser analisada no momento de coleta do material.

$\mathrm{Na}$ fase de propagação das mudas, o substrato deve apresentar características adequadas para promover maior capacidade de enraizamento e nutrição das mudas. Nesse sentido, o uso de biofertilizantes na formulação do substrato é uma prática sustentável. Sua 1*adição ao substrato pode melhorar os atributos químicos, físicos e microbiológicos (PRIETO et al., 2017), podendo contribuir no pegamento das mudas.

\footnotetext{
* 1 Trabalho baseado na Dissertação de Mestrado intitulada "Propagação vegetativa de Ora-pro-nóbis (Periskia aculeata Plum)". Disponível em: http://repositorio.ufgd.edu.br/jspui/handle/prefix/3153. Acesso em: $14 / 04 / 2021$.
} 
Dentre os biofertilizantes de base agroecológica, tanto comercialmente disponível bem como produzido, especialmente por agricultores familiares, tem-se o bokashi. É constituído de material de origem animal e/ou vegetal, fermentado por microrganismos benéficos, que podem disponibilizar nutrientes na forma orgânica de modo mais rápido em função do aumento da atividade microbiana (LIMA et al., 2015). No entanto, não há trabalhos na literatura associando esse biofertilizante à mudas de $P$. aculeata.

Considerando a importância da $P$. aculeata e o fato de que a adição de biofertilizante ao solo pode contribuir em maiores indicadores de brotação e desenvolvimento vegetativo da espécie, objetivou-se com este estudo conhecer o efeito do comprimento de estacas e do uso de biofertilizante bokashi na propagação vegetativa de $P$. aculeata por estaquia.

\section{MATERIAL E MÉTODOS}

O experimento foi realizado no período de junho a agosto de 2016, no Horto de

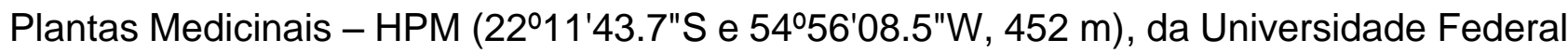
da Grande Dourados - UFGD, Dourados - MS, Brasil. As condições ambientais para a propagação das mudas foram as de viveiro coberto com tela de polipropileno de nylon com coloração preta, com retenção de $50 \%$ do fluxo de radiação solar.

Foram avaliados três comprimentos de estacas de $P$. aculeata $(15,20$ e $25 \mathrm{~cm})$, com diâmetro médio de $3,45 \mathrm{~mm}$, todos sem e com adição do biofertilizante bokashi ao substrato. $O$ delineamento experimental utilizado foi o de blocos casualizados, em que os tratamentos foram arranjados em esquema fatorial $3 \times 2$, com avaliações ao longo do tempo (parcelas subdivididas), com quatro repetições. A unidade experimental foi constituída por 10 recipientes transparentes plásticos de $500 \mathrm{~mL}$, cada um com uma estaca.

Para condução experimental, as estacas foram coletadas da região mediana de plantas matrizes mantidas a 25 anos no HPM da UFGD, as quais foram cortadas com tesoura de poda, no período matutino. Logo após, tiveram suas bases cortadas rentes ao nó basal e imersas em recipiente com água, para evitar a desidratação do tecido vegetal e a oxidação do nó basal.

O substrato base para a propagação da espécie foi composto por Latossolo Vermelho Distroférrico + $\operatorname{Tropstrato~}^{\circledR}(1: 1, \mathrm{v} / \mathrm{v})$, com os seguintes atributos químicos após a mistura, de acordo com a metodologia de Silva (2009): $\mathrm{pH} \mathrm{CaCl}_{2}=5,94 ; \mathrm{P}=42,65 \mathrm{mg} \mathrm{dm}^{3}$; $\mathrm{K}=1,99 \mathrm{cmol}_{\mathrm{c}} \mathrm{dm}^{3} ; \mathrm{Ca}=13,28 \mathrm{cmol}_{\mathrm{c}} \mathrm{dm}^{3}: \mathrm{Mg}=19,00 \mathrm{cmol}_{\mathrm{c}} \mathrm{dm}^{3} ; \mathrm{H}+\mathrm{Al}=2,37 \mathrm{cmol}_{\mathrm{c}} \mathrm{dm}^{3}$ 
e V $(\%)=62,88$. Os atributos químicos do bokashi (Garden Bokashi ${ }^{\circledR}$ ), segundo dados do fabricante, foram: $\mathrm{pH} \mathrm{CaCl}_{2}=6,1 ; \mathrm{N}=34,0 \mathrm{~g} \mathrm{~kg}^{-1} ; \mathrm{P}=8,0 \mathrm{~g} \mathrm{~kg}^{-1} ; \mathrm{K}=7,0 \mathrm{~g} \mathrm{~kg}^{-1} ; \mathrm{Ca}=22,0$ $\mathrm{g} \mathrm{kg}^{-1} ; \mathrm{Mg}=5,0 \mathrm{~g} \mathrm{~kg}^{-1}$; relação $\mathrm{C} / \mathrm{N}=11 / 1$; carbono orgânico $=400$. A dose utilizada foi de $10 \mathrm{~g} \mathrm{~kg}$ substrato $^{-1}$ de bokashi, nas parcelas com o uso do biofertizante.

O processo inicial de propagação consistiu no preenchimento dos recipientes com o substrato preparado, segundo a composição correspondente às unidades experimentais em estudo. Posteriormente as estacas foram enterradas no substrato de cada recipiente até a profundidade de $1 / 3$ da estaca.

Após o surgimento da primeira brotação, que ocorreu aos 21 dias após o enterrio das estacas - DAE, semanalmente, até 56 DAE, foram contadas as estacas com brotações, e depois calculadas a porcentagem de brotação utilizando-se a fórmula adaptada de Maguire (1946) Brot $=\frac{E \mathrm{~b}}{\mathrm{Ei}} \times 100$ (Equação 1) em que: Brot = brotações; $\mathrm{Eb}=$ número de estacas da parcela que tinham brotações e $\mathrm{Ei}=$ número de estacas implantadas por parcela. Também foi calculado o índice de velocidade de brotação $=\left[\operatorname{IVB}=n \div \sum \mathrm{ni}(1 \div\right.$ di)] (Equação 2) em que: $n$ = número de gemas brotadas; $n i$ = número de gemas brotadas na data i; di = dias até a brotação, adaptado de AMARAL (1979).

Aos $60 \mathrm{DAE}$, as mudas foram retiradas inteiras dos recipientes, lavadas e avaliadas quanto ao comprimento das raízes, número de brotos, folhas, raízes principais e enraizamento das estacas. Posteriormente, determinaram-se as áreas de superfície foliar e radicular das mudas utilizando-se integrador de área (LI-COR, Modelo $3100 \mathrm{C}$ - Área Meter, in Nebraska, USA). Também foram determinadas as massas frescas e secas, usando balança de precisão milesimal $\left(0,0001 \mathrm{~g}\right.$ - Modelo Shimadzu $\left.{ }^{\circledR}\right)$.

Para obtenção das massas secas, o material foi acondicionado em sacos de papel e submetidos à circulação forçada de ar em estufa a $60 \pm 5{ }^{\circ} \mathrm{C}$. Posteriormente, foram calculados os índices de razão de área foliar, massa foliar específica, peso foliar específico e relação raiz/parte aérea (Benincasa, 2003).

Os dados de brotação e índice de velocidade de brotação foram transformados em $\checkmark(x+1,0)$ para normalização. As médias dos dados relacionados à brotação e colheita foram submetidas à análise de variância (ANOVA), e quando significativos pelo teste $F$ ( $p$ $<0,05)$, as médias foram comparadas pelo teste t de Student, para bokashi, e Tukey, para comprimento de estacas $(p \leq 0,05)$. Os dados de porcentagem de brotação foram analisados em parcelas subdivididas no tempo, e submetidos à análise de variância, e de regressão $(p \leq 0,05)$. 


\section{RESULTADOS E DISCUSSÃO}

As porcentagens de brotação e de índice de velocidade de brotação das estacas de $P$. aculeata foram influenciadas pela interação comprimento de estacas e bokashi (Tabela 1). As maiores porcentagens de brotação e de índice de velocidade de brotação foram observadas nas estacas de $20 \mathrm{~cm}$ propagadas em substrato com bokashi (Tabela 1). Esses resultados mostram que as estacas precisam de quantidades equilibradas de substâncias energéticas de reservas e que confirma-se com as menores diferenças em relação às estacas com $25 \mathrm{~cm}$ e as propagadas sem bokashi.

Além disso, os menores valores tanto de brotação quanto do IVB ao utilizar estacas de $15 \mathrm{~cm}$, com bokashi ao substrato podem ser explicados pelo fato de nessa condição, ocorrer maior gasto energético, já que essas estacas apresentam menor quantidade de reservas e possivelmente fitohormônios, e também precisam absorver os nutrientes presentes no bokashi, reforçando a ideia de se conhecer o tamanho ideal das estacas para essa espécie.

O comprimento da estaca é importante para o desenvolvimento radicular, já que estacas maiores apresentam elevada quantidade de reservas nutritivas as quais podem ser translocadas para a base e auxiliar no enraizamento resultando em maior absorção de água e nutrientes favorecendo as brotações (PAULUS et al., 2014).

Tabela 1 - Brotação e índice de velocidade de brotação (IVB) em mudas de $P$. aculeata propagadas com diferentes comprimentos de estacas, sem e com bokashi ao substrato.

\begin{tabular}{ccccc}
\hline & \multicolumn{3}{c}{ Brotação (\%) } & \multicolumn{3}{c}{ IVB } \\
\hline \multirow{2}{*}{ Comprimento } & \multicolumn{5}{c}{ Com } & Sem & Com \\
\cline { 2 - 5 } & $55,0 \mathrm{aA}$ & $44,0 \mathrm{bB}$ & $0,7753 \mathrm{aA}$ & $0,6728 \mathrm{bA}$ \\
$15 \mathrm{~cm}$ & $46,0 \mathrm{aB}$ & $60,0 \mathrm{aA}$ & $0,6729 \mathrm{aB}$ & $0,8737 \mathrm{aA}$ \\
$20 \mathrm{~cm}$ & $55,0 \mathrm{aA}$ & $50,0 \mathrm{abA}$ & $0,7403 \mathrm{aA}$ & $0,7035 \mathrm{abA}$ \\
$25 \mathrm{~cm}$ & \multicolumn{4}{c}{13,74} \\
\hline C.V. (\%) & \multicolumn{3}{c}{19,43} &
\end{tabular}

Médias seguidas por letras minúsculas iguais, nas colunas, para comprimento de estacas, e maiúsculas nas linhas, para bokashi, não diferem entre si pelos testes de Tukey, e t de Student, respectivamente $(p>0,05)$.

Fonte: Os autores.

A porcentagem de brotação também foi influenciada de forma isolada pelas épocas de avaliação (Figura 1) observando-se crescimento linear, com $82 \%$ de brotação das estacas, aos 56 dias após a estaquia. Esse resultado deve-se a utilização gradual das reservas e metabolização dos hormônios para formação de brotação das estacas, assim 
como observado na formação de brotações em estacas de Hibiscus rosa-sinensis L. (PIZZATTO et al., 2011).

Figura 1 - Brotação de estacas de $P$. aculeata em função dos dias após a estaquia. * $(p<0,05)$.

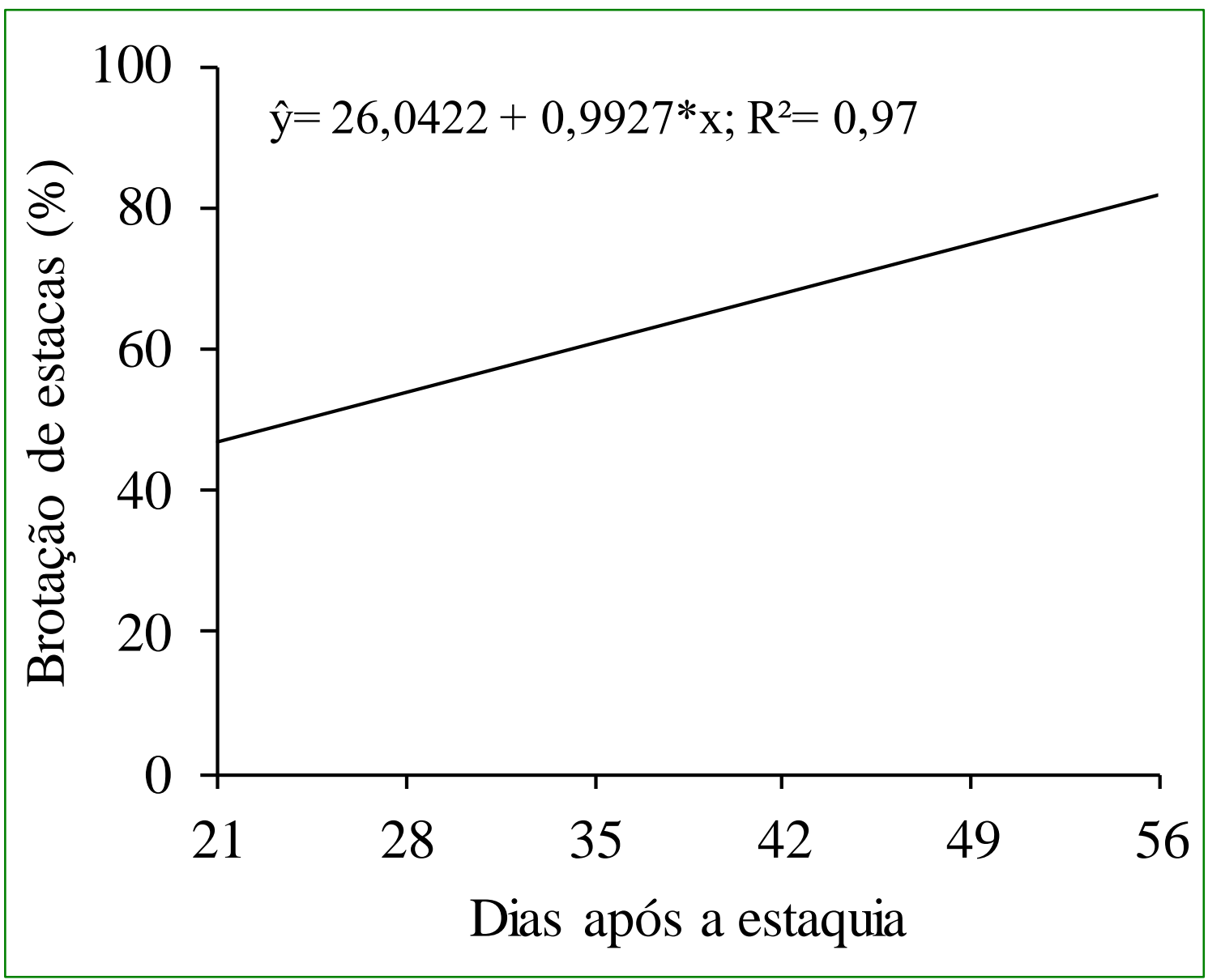

Fonte: Os autores.

O maior número de brotos por estaca ocorreu nas mudas procedentes de estacas de $20 \mathrm{~cm}$, sem adição de bokashi. Essa resposta é interessante, pois para obtenção de mudas com mais brotos, não há necessidade de custo com bokashi, e ainda consegue gerar mais unidade propagativas de uma planta matriz, pois a estaca é menor. Por outro lado, o maior NF e de área foliar ocorreram nas estacas com $25 \mathrm{~cm}$ com bokashi não diferindo estatisticamente do tratamento sem bokashi (Tabela 2). Esses resultados podem estar associados ao fato de que por essas estacas serem maiores, há maior quantidade de gemas e reservas, o que favorece essas características de crescimento.

De maneira geral, a avaliação da área foliar é importante, pois está relacionada à produção final de massa seca, uma vez que durante o ciclo de desenvolvimento, a planta 
depende tanto da taxa de expansão da AF como da taxa de fotossíntese por unidade de área foliar (MATOS et al., 2015), favorecendo maior produção de massas das folhas (Tabela 3).

A maior área radicular constatada nas estacas de $25 \mathrm{~cm}$, sem bokashi (Tabela 2) deve-se possivelmente de nessa condição haver maior necessidade de absorção de água e nutrientes. Nesse caso, as mudas apresentaram modificações quanto ao sistema radicular, visando otimizar área de exploração para aumentar a capacidade de interceptação de água e nutrientes (MIKKELSEN, 2015).

Por outro lado, ao adicionar o bokashi, ocorre melhorias nos aspectos microbiológicos, físicos e fertilidade do substrato (LIMA et al., 2015) na área rizosférica, e então as raízes apresentam menor área radicular por essas mudas estarem em boas condições de cultivo.

Tabela 2 - Número de brotos por estaca, folhas por broto, área foliar e radicular de mudas de $P$. aculeata propagadas com diferentes comprimentos de estacas, sem e com bokashi ao substrato.

\begin{tabular}{|c|c|c|c|c|}
\hline \multirow[b]{3}{*}{ Comprimento } & \multicolumn{2}{|c|}{ Número de brotos } & \multicolumn{2}{|c|}{ Número de folhas } \\
\hline & \multicolumn{4}{|c|}{ Bokashi } \\
\hline & Sem & Com & Sem & Com \\
\hline $15 \mathrm{~cm}$ & $4,83 \mathrm{bA}$ & $3,00 \mathrm{bB}$ & $15,33 \mathrm{bA}$ & $12,33 \mathrm{bA}$ \\
\hline $20 \mathrm{~cm}$ & $6,75 \mathrm{aA}$ & $3,87 \mathrm{bB}$ & $17,50 \mathrm{abA}$ & $12,50 \mathrm{bB}$ \\
\hline $25 \mathrm{~cm}$ & $5,12 \mathrm{aA}$ & $6,12 \mathrm{aA}$ & $21,25 \mathrm{aA}$ & $24,83 \mathrm{aA}$ \\
\hline \multirow[t]{3}{*}{ C.V. (\%) } & \multicolumn{2}{|c|}{14,38} & \multicolumn{2}{|c|}{14,90} \\
\hline & \multicolumn{2}{|c|}{ Área Foliar } & \multicolumn{2}{|c|}{ Área Radicular } \\
\hline & \multicolumn{4}{|c|}{ (cm²/planta) } \\
\hline \multirow{2}{*}{ Comprimento } & \multicolumn{4}{|c|}{ Bokashi } \\
\hline & Sem & Com & Sem & Com \\
\hline $15 \mathrm{~cm}$ & $66,00 \mathrm{bB}$ & $109,75 \mathrm{bA}$ & $12,66 \mathrm{bA}$ & $12,75 \mathrm{aA}$ \\
\hline $20 \mathrm{~cm}$ & $63,50 \mathrm{bA}$ & $75,33 \mathrm{cA}$ & $12,83 \mathrm{bA}$ & $15,83 \mathrm{aA}$ \\
\hline $25 \mathrm{~cm}$ & $141,33 \mathrm{aB}$ & $197,05 \mathrm{aA}$ & $22,16 \mathrm{aA}$ & $15,67 \mathrm{aB}$ \\
\hline
\end{tabular}

Médias seguidas por letras minúsculas iguais, nas colunas, para comprimento de estacas, e maiúsculas nas linhas, para bokashi, não diferem entre si pelos testes de Tukey, e t de Student, respectivamente $(p>0,05)$.

Fonte: Os autores.

O enraizamento e número de raízes não foram influenciados pelos fatores em estudo (Tabela 3), podendo ser justificado devido $P$. aculeata ser uma planta rústica, de fácil cultivo (TOFANELI \& RESENDE, 2011), além disso, apresenta rizogênese direta (ZEM et al., 2016), favorecendo o enraizamento, tal como observado neste estudo.

O maior comprimento das raízes e produção de massas frescas e secas das brotações nas estacas de $25 \mathrm{~cm}$ (Tabela 3) deve-se a maior quantidade de reservas 
armazenadas, tal como os carboidratos. Os carboidratos são fontes de carbono para a biossíntese de ácidos nucléicos, proteínas, além de recursos energéticos (CUNHA et al., 2009), favorecendo o fluxo de fotoassimilados utilizados na formação das brotações e raízes (OLIVEIRA et al., 2012).

Quanto às maiores massas secas das brotações com bokashi, pode estar relacionada à participação dos nutrientes disponibilizados em função da maior ciclagem devido ao aumento da atividade microbiana no substrato. Isso, porque o bokashi favorece a presença de microrganismos benéficos que atuam na ciclagem de nutrientes e dinâmica da decomposição (ALVAREZ-SOLíS et al., 2016), disponibilizando os nutrientes para o desenvolvimento das plantas, dentre eles o nitrogênio. O nitrogênio está associado a síntese de proteínas e de ácidos nucleicos (CUNHA et al., 2009), sendo responsável pela desdiferenciação dos meristemas radiculares e apicais (PICOLOTTO et al., 2015).

Tabela 3 - Estacas enraizadas (EE), número das maiores raízes principais (NRP), comprimento da maior raiz (CR) e massa fresca (MFB) e secas (MSB) das brotações de mudas de $P$. aculeata propagadas com diferentes comprimentos de estacas, sem e com bokashi ao substrato.

\begin{tabular}{cccccc}
\hline & EE & NRP & CR & MFB & MSB \\
\hline Bokashi & $(\%)$ & (unid.) & $(\mathrm{cm})$ & \multicolumn{2}{c}{ (g/planta) } \\
\hline Sem & $91 \mathrm{a}$ & $2,75 \mathrm{a}$ & $14,14 \mathrm{a}$ & $13,84 \mathrm{a}$ & $6,72 \mathrm{~b}$ \\
Com & $96 \mathrm{a}$ & $2,37 \mathrm{a}$ & $13,05 \mathrm{a}$ & $15,30 \mathrm{a}$ & $9,18 \mathrm{a}$ \\
\hline Comprimento & & & & & \\
\hline $15 \mathrm{~cm}$ & $86 \mathrm{a}$ & $1,87 \mathrm{a}$ & $11,30 \mathrm{~b}$ & $11,40 \mathrm{~b}$ & $3,28 \mathrm{~b}$ \\
$20 \mathrm{~cm}$ & $100 \mathrm{a}$ & $2,93 \mathrm{a}$ & $12,90 \mathrm{~b}$ & $13,07 \mathrm{~b}$ & $5,95 \mathrm{~b}$ \\
$25 \mathrm{~cm}$ & $94 \mathrm{a}$ & $2,87 \mathrm{a}$ & $16,58 \mathrm{a}$ & $19,23 \mathrm{a}$ & $11,22 \mathrm{a}$ \\
\hline C.V. $(\%)$ & 14,61 & 48,00 & 17,28 & 20,70 & 23,89 \\
\hline
\end{tabular}

Médias seguidas por letras minúsculas iguais, nas colunas, não diferem entre si, para bokashi, pelo teste t de Student, e para comprimento de estacas, pelo teste de Tukey $(p>0,05)$.

Fonte: Os autores.

Em mudas de mamoeiro, avaliando-se doses de bokashi (0, 3, 6 e 10\%), constatouse que adição de $6 \%$ favoreceu incremento no número de folhas $(14,95)$, comprimento da parte aérea $(30,29 \mathrm{~cm})$ e das raízes $(25,28 \mathrm{~cm})$ e produção de massa seca total $(2.176,8 \mathrm{~g})$ (HAFLE et al., 2009). Esses resultados vêm de encontro com o que ocorreu nesse experimento com relação ao incremento proporcionado pelo bokashi.

As maiores massas secas e frescas de raízes foram observadas em estacas de 25 cm, sem bokashi (Tabela 4), de maneira similar aos resultados de área radicular (Tabela 2), fato que favoreceu a absorção de água e nutrientes. A maior relação $\mathrm{R} / \mathrm{Pa}$ ocorreu nas mudas provenientes de estacas de $20 \mathrm{~cm}$, com adição de bokashi (Tabela 4) devido à maior 
exportação de fotoassimilados entre esses órgãos. Sugere-se que quando se obtêm mudas provenientes dessas estacas, há maior estabilidade do desenvolvimento.

Tabela 4 - Massas frescas (MFR) e secas (MSR) de raízes de mudas de $P$. aculeata propagadas com diferentes comprimentos de estacas, sem e com bokashi ao substrato.

\begin{tabular}{ccccc}
\hline & \multicolumn{3}{c}{ MFR } & \multicolumn{2}{c}{ MSR } \\
\hline \multirow{2}{*}{ Comprimento } & \multicolumn{5}{c}{ Bokashi } \\
\cline { 2 - 5 } & Sem & Com & Sem & Com \\
\cline { 2 - 5 } & $1,73 \mathrm{bA}$ & $1,96 \mathrm{aA}$ & $0,53 \mathrm{bA}$ & $0,79 \mathrm{aA}$ \\
$20 \mathrm{~cm}$ & $1,81 \mathrm{bB}$ & $2,84 \mathrm{aA}$ & $0,61 \mathrm{bB}$ & $1,54 \mathrm{aA}$ \\
$25 \mathrm{~cm}$ & $3,58 \mathrm{aA}$ & $2,59 \mathrm{aB}$ & $2,38 \mathrm{aA}$ & $1,64 \mathrm{aA}$ \\
\hline C.V. $(\%)$ & & 26,53 & & 37,00 \\
\hline
\end{tabular}

Médias seguidas por letras minúsculas iguais, nas colunas, para comprimento de estacas, e maiúsculas nas linhas, para bokashi, não diferem entre si pelos testes de Tukey, e t de Student, respectivamente $(p>0,05)$ Fonte: Os autores.

Constatou-se maior razão de massa foliar nas mudas provenientes de estacas de 25 $\mathrm{cm}$, com bokashi (Tabela 6), provavelmente deve-se a maior área foliar obtida neste mesmo tratamento (Tabela 2). Isso pode ser explicado pelo fato de que essa característica representa a capacidade de translocação de fotoassimilados da parte aérea para o resto da planta e, quanto maior for esta razão, mais eficiente é a translocação (SCALON et al., 2006). Neste caso, a maior área foliar favoreceu a interceptação de radiação solar e conversão em energia química e produção de fotoassimilados para a planta.

A razão de área foliar não foi influenciada pelos fatores em estudo (Tabela 6); já a maior massa foliar específica foi das mudas das estacas de $20 \mathrm{~cm}$, independendo do uso de bokashi em função das maiores características de crescimento da área foliar (Tabela 2) e massa seca das mudas (Tabela 3), o que pode estar associado a quantidade de gemas das estacas a partir desse tamanho. A massa foliar específica expressa a fração de massa seca retida na planta e a área foliar, isto é, a medida que a planta expressa potencial em crescimento, maior é a exportação de fotoassimilados das folhas para os outros órgãos (BENINCASA, 2003). 
Tabela 5 - Relação de massa da raiz/parte aérea (R/Pa) e razão de massa foliar (RMF) de mudas de $P$. aculeata propagadas com diferentes comprimentos de estacas, sem e com bokashi ao substrato.

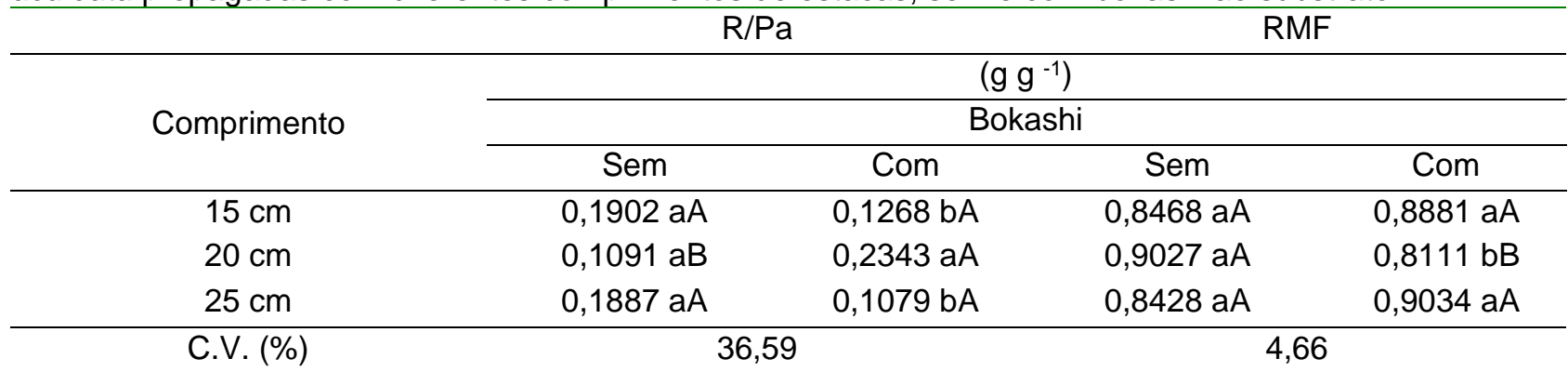

Médias seguidas por letras minúsculas iguais, nas colunas, para comprimento de estacas, e maiúsculas nas linhas, para bokashi, não diferem entre si pelos testes de Tukey e t de Student, respectivamente $(p>0,05)$.

Fonte: Os autores.

Tabela 6 - Razão de área foliar (RAF) e massa foliar específica (MFE) em mudas de $P$. aculeata em função do comprimento de estacas, sem e com bokashi ao substrato.

\begin{tabular}{ccc}
\hline & RAF & MFE \\
\hline Bokashi & $\left(\mathrm{cm}^{2} \mathrm{~g}^{-1}\right)$ & $\left(\mathrm{g} \mathrm{cm}^{-2}\right)$ \\
\hline Sem & $8,8792 \mathrm{a}$ & $0,1248 \mathrm{a}$ \\
Com & $8,4608 \mathrm{a}$ & $0,1059 \mathrm{a}$ \\
\hline Comprimento & & $0,0855 \mathrm{~b}$ \\
\hline $15 \mathrm{~cm}$ & $10,2700 \mathrm{a}$ & $0,1578 \mathrm{a}$ \\
$20 \mathrm{~cm}$ & $7,1519 \mathrm{a}$ & $0,1029 \mathrm{ab}$ \\
\hline C.V. $(\%)$ & $8,5881 \mathrm{a}$ & 41,83 \\
\hline
\end{tabular}

Médias seguidas por letras minúsculas iguais, nas colunas, não diferem entre si, para bokashi, pelo teste t de Student, e para comprimento de estacas, pelo teste de Tukey $(p>0,05)$.

Fonte: Os autores.

As informações obtidas neste trabalho são imprescindíveis na disseminação de técnicas de propagação para $P$. aculeata, tendo em vista sua importância alimentícia e medicinal, isto é, com potencial de inserção na cadeia produtiva visando agregação de valor à produção vegetal, bem como valorização e consumo das PANC's.Todavia, salienta-se que as pesquisas utilizando biofertilizantes na formulação de substratos são incipientes, tornando-se necessário desenvolvimento de pesquisas futuras que identifiquem quantidades adequadas para produção de mudas. 


\section{CONCLUSÕES}

Para propagação $P$. aculeata recomenda-se utilizar estacas de $20 \mathrm{~cm}$ com a adição de bokashi ao substrato por promover maior porcentagem de brotação e índice de velocidade de brotação. No que se refere às características de crescimento, foram maiores nas mudas provenientes das estacas de $25 \mathrm{~cm}$, independente do uso do bokashi.

\section{REFERÊNCIAS}

ALMEIDA, M. E. F; CORRÊA, A. D. Utilização de cactáceas do gênero Pereskia na alimentação humana em um município de Minas Gerais. Ciência Rural, v. 42, n. 4, p. 751756, 2012.

ÁLVAREZ-SOLÍS, J. D.; MENDONZA-NÚNES, J. A.; NOE, S.; LEÓN-MARTINÉS, N. S. L.; CASTELLANOS-ALBORES. J.; GUTIÉRRES-MICELI, F. A. Effect of bokashi and vermicompost leachate on yield and quality of pepper (Capsicum annuum) and onion (Allium сера) under monoculture and intercropping cultures. Ciencia e Investigación Agraria, v. 4, n. 2, p. 243-252, 2016.

AMARAL E. Alguns problemas de estatística aplicada em análise de sementes. Tecnologia de Sementes, v. 2, n. 1, p. I2-18, 1979.

BENICASA, M. M. P. Análise do crescimento de plantas (noções básicas). Jaboticabal: FUNEP, 2003. $41 \mathrm{P}$.

CARDOSO, A. I. I. Características foliares e índice de colheita do rabanete irrigado com agua ressudariam em ambiente protegido. Enciclopédia Biosfera, Centro Científico Conhecer, v. 1, n. 21, p. 375-386, 2015.

CUNHA, A. C. M.; PAIVA, H. N.; XAVIER, A.; OTONI, W. C. Papel da nutrição mineral na formação de raízes adventícias em plantas lenhosas. Pesquisa Florestal Brasileira, v. 58, n. 58, p. 35-45, 2009.

HAFLE, O. M.; SANTOS, V. A.; RAMOS, J. D.; CRUZ, M. C. M.; MELO, P. C. Produção de mudas de mamoeiro utilizando bokashi e lithothamnium. Revista Brasileira de Fruticultura, v. 31, n. 1, p. 245-251, 2009.

KINUPP, V. F.; LORENZI, H. Plantas alimentícias não convencionais (PANC) no Brasil: guia de identificação, aspectos nutricionais e receitas ilustradas. São Paulo: Instituto Plantarum de Estudos da Flora, 2014. 768p. 
LIMA, C. E. P.; FONTENELlE, M. R.; SILVA, L. B.; SOARES, D. C.; MOITA, A. W.; ZANDONANI, D. B.; SOUZA, R. B.; LOPES, C. A. Short-term changes in fertility attributes and soil organic matter caused by the addition of EM bokashis in two tropical soils. International Journal Agronomy, v. 2015, n. 754298, p. 1-9, 2015.

MAGUIRE, J. D. Speed of germination aid in selection and evaluation for seedling emergence and vigor. Crop Science, v. 2, n. 1, p. 176-177, 1962.

MIKKELSEN, R. (2015) - Ano internacional dos solos: edição especial. Informe Agronômico, ำ 150. International Plant Nutrition Institute (IPNI). http://ipni.net/publication/iabrasil.nsf, acesso em 23 ago. 2017.

OLIVEIRA, R. J. P.; BIACHI, V. J.; AIRES, R. F.; CAMPOS, A. D. Teores de carboidratos em estacas lenhosas de mirtileiro. Revista Brasileira de Fruticultura, v. 34, n. 4, p. 11991207, 2012.

PAULUS, D.; VALMORBIDA, R.; TOFFOLI, E.; PAULUS, E. Propagação vegetativa de Aloysia triphylla (L'Hér.) Britton em função da concentração da AIB e do comprimento das estacas. Revista Brasileira de Plantas Medicinais, v. 16, n. 1, p. 25-31, 2014.

PICOLOTTO, L.; VIGNOLO, G K.; PEREIRA, I. S.; GONÇALVES, M. A.; ANTUNES, L. A. C. Enraizamento de estacas de amoreira-preta em função da adubação nitrogenada na planta matriz. Revista Ceres, v. 62, n. 3, p. 294-300, 2015.

PIZZATO, M.; JÚNIOR, A. W.; LUCKMANN, D.; PIROLA, K.; CASSOL, D. A.; MAZARO, S. M. Influência do uso de AIB, época de coleta e tamanho de estaca na propagação vegetativa de hibisco por estaquia. Revista Ceres, v. 58, n. 4, p. 487-492, 2011.

PRIETO, C. A.; AlVAREZ, J. W. R.; FIGUEIREDO, J. C. K.; TRINIDADE, S. A. Bioestimulante, biofertilizante e inoculação de sementes no crescimento e produtividade da soja. Revista Agricultura Neotropical, v. 4, n. 2, p. 1-8, 2017.

SCALON, S. P. Q.; MUSSURY, R. M.; SCALON FILHO, H.; FRANCELINO, C. S. F. Desenvolvimento de mudas de aroeira (Schinus terebinthifolius) e sombreiro (Clitoria fairchildiana) sob condições de sombreamento. Ciências Agrotecnologia, v. 30, n. 1, p. 166-169, 2006.

SILVA, F. C. Manual de análises químicas do solo, plantas e fertilizantes. 2. ed. rev. ampliada-Brasília, DF: Embrapa Informação Tecnológica, 2009. 627p.

SILVA, G. C.; OLIVEIRA, L. M.; LUCCHESE, A. M.; SILVA, T. R. S.; NASCIMENTO, M. N. Propagação vegetativa e crescimento inicial de Lippia origanoides (alecrim de tabuleiro). Horticultura Brasileira, v. 33, n. 2, p. 236-240, 2015.

SOUZA, M. R. DE M.; MILAGRES, C. S. F.; PEREIRA, R. G. F.; PINTO, C. L. DE O.; CAIXETA, G. Z. T.; PEREIRA, P. R. G. Perfil de produção e comercialização do ora-pronobis em dois contextos regionais de minas gerais: perspectivas de agregação de valor. Revista Brasileira de Agropecuária Sustentável, v. 6, n. 4, p. 45-50, 2016. 
SOUZA, M. S. S.; BARBALHO, S. M.; GUIGUER, E. L; ARAÚJO, A. C.; BUENO, P. C. S.; FARINAZZI-MACHADO, F. M. V.; LIMA, L. M. L.; SILVA, B. C.; MENDES, C. G. Effect os Pereskia aculeata Miller on the biochemical profiles and body composition of wistar rats. Journal Bioscience Medicines, v. 3, n. 7, p. 82-89, 2015.

SOUZA, R. M.; LIRA C. S.; RODRIGUES, A. O.; MORAIS, S. A. L; QUEIROZ, C. R. A. A; CHANG, R.; AQUINO, F. J. T; MUÑOZ, R. A. A; OLIVEIRA, A. Atividade antioxidante de extratos de folhas de ora pro nóbis (Pereskia aculeata Mill.) usando métodos espectrofotométricos e voltamétricos in vitro. Bioscience Journal, v. 30, n. 1, p. 448-457, 2014.

TOFANELLI, M. B. D.; RESENDE, S. G. Sistemas de condução na produção de folhas de Ora pro nóbis. Pesquisa Agropecuária Tropical, v. 41, n. 3, p.466-469, 2011.

ZEM, L. M.; ZUFFELLATO-RIBAS, K. C.; KOEHLER, H. S. Enraizamento de estacas semilenhosas de Pereskia aculeata nas quatro estações do ano em diferentes substratos. Revista Eletrônica Científica, v. 2, n. 3, p. 227-233, 2016. 


\section{ABSTRACT}

There is little technical information on the factors that can influence the vegetative propagation of the Pereskia aculeata Plum. Thus, the aim this study was to know the effect of the length of cuttings and the use of bokashi in the propagation of $P$. aculeata. Three lengths of cuttings were evaluated: 15,20 and $25 \mathrm{~cm}$, without and with bokashi, under nursery with $50 \%$ shade. The cuttings were propagated in $500 \mathrm{~mL}$ plastic containers with substrate Dystrophic Red Latosol + Tropstrato (1:1, v/v). Sprouts were evaluated weekly after the first sprouting, and at 60 days after cutting, whole seedlings were harvested. The highest sprouting percentage was $60 \%$ and the sprout velocity index of 0.8737 of the $20 \mathrm{~cm}$ cuttings, with bokashi. The highest number of sprout (6.12) and leaves (24.83) was observed in $25 \mathrm{~cm}$ cuttings, independent of use bokashi. The highest values root length $(16.58 \mathrm{~cm})$ and the production of fresh and dry mass sprouting (19.23 and $11.22 \mathrm{~g}$, respectively) were in seedlings with cuttings of $25 \mathrm{~cm}$. The addition of bokashi and cuttings $25 \mathrm{~cm}$ favored higher production of dry mass sprouts. Use of cuttings $25 \mathrm{~cm}$ contribute to the production of $P$. aculeata seedlings because they favor sprouting indicators, leaf area and physiological indices.

Keywords: Biofertilizers. Sprout. Cuttings. Medicinal plant. PANC's.

\section{RESUMEN}

Existen pocas informaciones técnicas sobre los factores que pueden influenciar en la propagación vegetativa de Pereskia acueata Plum. Así, se objetiva con esta investigación conocer el efecto de la altura de las estacas y el uso de bokashi en la propagación de $P$. aculeata. Se evaluaron tres alturas de estacas: 15,20 y $25 \mathrm{~cm}$, sin y con bokashi, en vivero con $50 \%$ de sombra. Las estacas fueron propagadas en recipientes plásticos de $500 \mathrm{~mL}$, con substrato Ferralsol Rojo Distroférrico + $\operatorname{Tropstrato~}^{\circledR}(1: 1, \mathrm{v} / \mathrm{v})$. Los brotes fueron evaluados semanalmente después del día del surgimiento del primer brote, y a los 60 días después de la estaquia, se cosecharon las plántulas enteras. El porcentaje más grande de brote fue de $60 \%$ y el índice de velocidad de brote de 0,8737 en las mudas provenientes de las estacas de $20 \mathrm{~cm}$ con bokashi. El número más grande de brotes $(6,12)$ y de hojas $(24,83)$ fue constatado en las estacas de $25 \mathrm{~cm}$, independiente del bokashi. Los valores más grandes del largo de las raíces $(16,58 \mathrm{~cm})$ y de producción de masas frescas y secas de los brotes (19,23 e 11,22 g, respectivamente) fueron en las estacas de $25 \mathrm{~cm}$. La adición de bokashi en las estacas de $25 \mathrm{~cm}$ fue favorable para una producción más grande de masa seca de brotes. El uso de estacas de $25 \mathrm{~cm}$ contribuyó en la producción de mudas de $P$. aculeata por favorecer los indicadores de brotes, área foliar e indicadores fisiológicos.

Palabras clave: Biofertilizante. Brotes. Estacas. Planta medicinal. PANC's. 


\section{LICENÇA DE USO}

Este é um artigo publicado em acesso aberto (Open Access) sob a licença Creative Commons Atribuição 4.0 Internacional (CC BY 4.0), que permite uso, distribuição e reprodução em qualquer meio, desde que o trabalho original seja corretamente citado. Mais informações em: http://creativecommons.org/licenses/by/4.0

\section{CONFLITO DE INTERESSES}

Os autores declaram que não há conflito de interesses neste trabalho.

\section{CONTRIBUIÇÕES AUTORAIS}

Juliana Milene Silverio: responsável pela condução experimental, coleta de dados, interpretação e redação científica.

Cleberton Correia Santos: responsável pelo planejamento, análises estatísticas, redação e revisão do manuscrito.

Venâncio Estevão Wilkomm: responsável pela condução experimental, coleta de tomadas de dados e redação científica.

Diogo Roberto Bruschis Karas: responsável pela condução experimental, coleta de dados e redação científica.

Maria do Carmo Vieira: responsável pela revisão do manuscrito.

Néstor Antonio Heredia Zárate: responsável pela revisão do manuscrito.

\section{FINANCIAMENTO}

Os autores agradecem ao Conselho Nacional de Desenvolvimento Científico e Tecnológico (CNPq) e Coordenação de Aperfeiçoamento de Pessoa de Nível Superior (CAPES), pela concessão de bolsas, e à Fundação de Apoio ao Desenvolvimento de Ensino, Ciência e Tecnologia do Mato Grosso do Sul (FUNDECT), pelo apoio financeiro.

\section{COMO REFERENCIAR}

SILVERIO, Juliana Milene; SANTOS, Cleberton Correia; WILKOMM, Venâncio Estevão; KARAS, Diogo Roberto Bruschis; VIEIRA, Maria do Carmo; ZÁRATE, Néstor Antonio Heredia. Bokashi e comprimento de estacas na propagação vegetativa de Pereskia aculeata plum (ora-pro-nóbis). Revista Brasileira de Engenharia de Biossistemas (Tupã), v. 15, n. 1, p. 90-104, 2021. DOI: http://dx.doi.org/10.18011/bioeng2021v15n1p90104.

\section{RESPONSABILIBADE EDITORIAL}

Prof. Dr. Fernando Ferrari Putti ${ }^{1}$, Prof. Dr. Paulo Sérgio Barbosa dos Santos ${ }^{1}$, Prof. Dr. Eduardo Festozo Vicente ${ }^{1}$ e Prof. Dr. Diogo de Lucca Sartori ${ }^{1}$

${ }^{1}$ Universidade Estadual Paulista "Júlio de Mesquita Filho", FCE - Faculdade de Ciências e Engenharia, Tupã, SP, Brasil. 